\title{
Mechanism of Vortices Appearance in the Taylor-Couette Flow System
}

\author{
M. Khirennas ${ }^{1 \dagger}$, H. Oualli ${ }^{1}$, M. Mekadem ${ }^{1}$, T. Azzam ${ }^{1}$, A. Benaiche ${ }^{1}$ and A. Bouabdallah ${ }^{2}$ \\ ${ }^{1}$ Laboratory of Fluid Mechanics, Military Polytechnical school, Bordj El Bahri, 16046 Alger. Algeria \\ ${ }^{2}$ Laboratory of Thermodynamics and Energetic Systems, USTHB, Bp 32 El-Alia, Alger, 16111, Algeria
}

$\dagger$ Corresponding Author Email: khirennasmessaoudtc@gmail.com

(Received February 8, 2021; accepted August 9, 2021)

\begin{abstract}
This work is devoted to study the Taylor-Couette flow at the early structuring stages. It is aimed to gain insight on the Taylor and Ekman vortices genesis mechanism since the first hints of presence detected at $\mathrm{Ta}=10^{-4}$. Simulations are carried out using Ansys Fluent software package. The basic system geometry is characterized bya height $\mathrm{H}=150 \mathrm{~mm}$, ratio of inner to outer cylinder radii $\eta=0.9$, radial gap $\delta=0.11$ and an aspect ratio corresponding to system height reported togap length, $\Gamma=\mathrm{H} / \delta=15$. Ekman and Taylor cells are tackled since the Taylor number $\mathrm{Ta}=10^{-4}$ to the first (TVF) and second (WVF) instabilities settlement at $\mathrm{Ta}_{\mathrm{c}}=43.8$ and $\mathrm{Ta}_{\mathrm{c} 2}=$ 54 , respectively. It is sought to shed light on the underlying mechanism responsible for flow genesis and to identify all the intermediate successive steps from ex-nihilo when the system is at rest up to complete vortices formation. The obtained results show that presence of Ekman cells is already perceptible since a Taylor number as low as $\mathrm{Ta}=10^{-4}$. In fact, localized overpressure zones are detected on system inner endcaps surfaces regularly distributed according to a $\pi / 2$ phase lag. These overpressure zones azimuthally propagate to meet and cover the entire gap circumference when Ta $\sim 10^{-2}$ to $10^{-1}$.
\end{abstract}

Keywords: CFD; Taylor-Couette flow; Ekman cells; Fluent software; TVF; WVF.

\section{NOMENCLATURE}

$H \quad$ height of cylinder

$R_{1} \quad$ radius of innercylinder

$R_{2} \quad$ radius of outer cylinder

$\mathrm{Ta}$ Taylor number

$T a_{c l} \quad$ first instability critical taylor

$\mathrm{Ta}_{c 2} \quad$ second instability critical taylor

$C C F \quad$ Circular Couette Flow

\section{INTRODUCTION}

Rotating flows are thoroughly considered in literature devoted to applied sciences. This is certainly due to diversity of the fundamental and applied aspects with the wealthy involved phenomenology exhibited particularly when laminar-turbulent transition is considered.

The Taylor-Couette flow is defined as a fluid movement evolving in the annular gap of two concentric cylinders with one or both are set in rotation or counter-rotation. Several applications are relevant to this flow system in industry such as turbomachinery, mixing processes, tribology, tangential filtration, crystallization, etc. It is also

$\begin{array}{ll}T V F & \text { Taylor Vortex Flow } \\ W V F & \text { Wavy Vortex Flow } \\ \eta & \text { radius ratio }=\mathrm{R}_{1} / \mathrm{R}_{2} \\ \Omega_{l} & \text { angular velocity of inner cylinder } \\ \delta & \text { gap width }=\mathrm{R}_{2}-\mathrm{R}_{1} \\ \Gamma & \text { aspect ratio }=\mathrm{H} / \delta \\ v & \text { kinematic viscosity }\end{array}$

used in nuclear reactors tours insulation, heat exchangers and in hydrocarbon drilling. Since the pioneering work of Couette (1890) and Taylor (1923), the rotor-stator configuration is subject to tremendous continuous researching studies.

As pioneers, Stockes (1848) used two concentric cylinders to show that rotation of the inner cylinder should lead to unstable flow. In (1890), Couette inspired the work of Margules (1881) to develop the first viscometer. Later, Rayleigh (1916). studied the behavior and stability of inviscid rotating flows to define the first stability criterion for the case of rotating cylinders. In1923, Taylor considered both analytically and experimentally the flow evolving between two concentric cylinders. The study focused 
on the vortices appearance when the inner cylinder rotating velocity is progressively increased. Coles (1965) presented the first experimental investigation showing existence of various flow patterns in the Taylor-Couette flow. Gollub and Swinney (1975) carried out an important experimental study and reported the important result that a succession of only few instabilities is sufficient to drive the flow from stability to chaoticity. In 1979, Fenstermacher and Swinney used laser-doppler velocimetry technique to study transition to turbulence of a fluid contained between concentric cylinders with a rotating inner cylinder. Bouabdallah (1980) presented an analytical and experimental study for various transition regimes from laminar to fully developed turbulence. Using chaos theory, he found that the critical value of the Taylor number is $T_{a c l}=$ 41.2. This is adopted as one of the referential studies for results validation herein. Czarny et al. (2003) investigated the interaction between Ekman pumping and the centrifugal instability in TaylorCouette flow-considering three endwall boundary conditions to analyse the nature of these interactions. Further Czarny et al. (2004) investigated the interaction between the endwall Ekman cells and the vortical structures in a finite-length cavity with differentially rotating concentric cylinders using Direct Numerical Simulations (DNS) with a threedimensional spectral method. It is reported that thickness and strength of Ekman layers at the endwalls match well with those predicted from a simple theoretical approach. Ahlers and Cannell (1983), Lücke (1985) and Pfister and Rehberg (1981) considered a circular Couette flow transition and patterns formation of Taylor vortices growing into unstable such a flow. It is established that below a critical Reynolds number (Re) characterizing transition from non-vortical to vortical flow, vortices near endwalls, recognized as Ekman vortices, are driven by the boundary flow. These endwall vortices are responsible in turn on driving adjacent axially piled Taylor vortices. At the current stage, all researches are carried out using active control of Taylor-Couette flow, to delay or advance appearance of the first bifurcation and other structures. It is essentially noted that axial oscillation of the inner cylinder is used as a stabilizing effect on the Taylor vortex flow. Ludwieg (1964) considered oscillation effect on Taylor vortices onset and attracted potential interest on both fundamental and applied aspects. Axial oscillation is used as a stabilizing strategy leading to retarding triggering of the first instability at high Taylor numbers.

Serre et al. (2008) studied Stability of TaylorCouette flow in a finite-length cavity with radial through flow. It is concluded that this radial through flow alters the stability of the flow system in such a way that a stabilizing effect is obtained by radial inflow and outflow. A slightly destabilizing effect is achieved by weak radial outflow. Goharzadeh and Mutabazi (2001) experimentally characterized flow intermittency regimes. Czarny et al. (2002) tackled spiral and wavy vortex flows in short counterrotating Taylor-Couette cell. Denis et al. (2014) developed a technique that seems to be able to characterize transition mechanism responsible of driving Taylor-Couette flow to waviness patterns.

More recently, Oualli et al. (2013) numerically investigated effect of the outer cylinder radial oscillation on the laminar-turbulence transition in infinite length cylinders. They found that this control strategy had a stabilizing effect on the evolving flow and the first flow bifurcation is delayed to a Taylor number $\mathrm{Ta}_{\mathrm{c} 1}=70$ for a deforming amplitude of $15 \%$ the external cylinder diameter value. Abdelali et al. (2019) investigated first instabilities onset delay using a controlling strategy based upon a combination of inner cylinder cross-section oscillation with a free surface effect. Two ranges of deformation frequencies are considered, lower frequencies, $\mathrm{f}<3 \mathrm{~Hz}$ to destroy Taylor vortices, and higher frequencies, $\mathrm{f}>20 \mathrm{~Hz}$ to make Ekman cells, identified as more resistant, completely disappearing.

In this paper, a numerical study is elaborated on the Taylor-Couette flow. The angular velocity is progressively increased from a Taylor number $\mathrm{Ta}=$ $10^{-4}$ to $\mathrm{Ta}_{\mathrm{c} 1}=43.8(\mathrm{TVF})$ and $\mathrm{Ta}_{\mathrm{c} 2}=54(\mathrm{WVF})$ in order to follow the flow structuring mechanism from nascent Ekman cells to fully developed Taylor regime. The first instability settlement is found at the critical Taylor number, $\mathrm{Ta}_{\mathrm{c} 1}=43.8$ and the second at $\mathrm{Ta}_{\mathrm{c} 2}=54$ corresponding respectively to Taylor Vortex Flow (TVF) and Wavy mode.

\section{GOVERNING EQUATIONS}

A viscous fluid confined between two concentric cylinders of length $\mathrm{H}$, with inner and outer radii respectively, $R_{1}$ and $R_{2}$ is considered. The angular velocity of the inner cylinder rotation rate is $\Omega_{1}$, while the outer as well as the top and bottom endwalls are maintained at rest, as shown in Fig.1. The flow is governed by three dimensionless parameters, namely, the inner to outer radii ratio $\eta=$ $\mathrm{R}_{1} / \mathrm{R}_{2}$, the aspect ratio $\Gamma=\mathrm{H} / \delta$ corresponding to the cylinder height reported to the gap length, and the Taylor number defined as:

$T a=\frac{\Omega 1 \cdot R 1 \cdot \delta 1}{v \sqrt{ }\left(\frac{\delta}{R 1}\right)}$

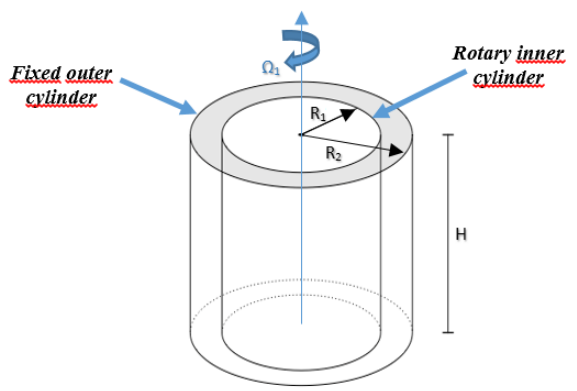

Fig. 1. Schematic of the Taylor-Couette flow system.

A numerical simulation of such a flow motion is implemented using Navier-Stokes equations based upon the classical physical laws of conservation, continuity and momentum equations for an 
incompressible fluid flow written in a cylindrical coordinates reference $(\mathrm{r}, \theta, \mathrm{z})$ as follows:

- Continuity equation :

$\frac{1}{r} \frac{\partial}{\partial r}(r \cdot U)+\frac{1}{r} \frac{\partial V}{\partial \theta}+\frac{\partial W}{\partial z}=0$

- Momentum equations :

$r: \frac{D}{D t} U-\frac{V^{2}}{r}=-\frac{1}{\rho} \frac{\partial P}{\partial r}+V\left[\left(\nabla^{2}-\frac{1}{r^{2}}\right) U\right.$

$$
\left.-\frac{2}{r^{2}} \frac{\partial V}{\partial \theta}\right]
$$

$\theta: \frac{D}{D t} V+U \frac{V}{r}=-\frac{1}{\rho} \frac{1}{r} \frac{\partial P}{\partial \theta}$

$$
+V\left[\left(\nabla^{2}-\frac{1}{r}\right) \cdot V+\frac{2}{r^{2}} \frac{\partial U}{\partial \theta}\right]
$$

$z: \frac{D}{D t} W=-\frac{1}{\rho} \frac{\partial P}{\partial z}+V \cdot \nabla^{2} \cdot W$

The symbol (D/Dt) stands for differential operator representing total derivative, such as:

$\frac{D}{D t}=\frac{\partial}{\partial t}+U \frac{\partial}{\partial r}+\frac{V}{r} \frac{\partial}{\partial \theta}+W \frac{\partial}{\partial z}$

Notation $\Delta=\nabla^{2}$ stands for Laplacian operator in $(\mathrm{r}, \theta, \mathrm{z})$ coordinates:

$\nabla^{2}=\frac{\partial^{2}}{\partial r^{2}}+\frac{1}{r} \frac{\partial}{\partial r}+\frac{1}{r^{2}} \frac{\partial^{2}}{\partial \theta^{2}}+\frac{\partial^{2}}{\partial z^{2}}$

$(\mathrm{U}, \mathrm{V}, \mathrm{W})$ are velocity components and $\mathrm{P}$ is the pressure.

\section{BOUNDARY CONDITIONS}

In this study, the Taylor-Couette system is considered in such a way that the fluid is driven by the rotating inner cylinder, while the outer with the upper and lower endwalls are maintained fixed. The corresponding boundary conditions are thus: inner cylinder)

$$
\mathrm{r}=\mathrm{R}_{1}: \mathrm{V}=\mathrm{R}_{1} \Omega_{1} \text { and } \mathrm{U}=\mathrm{W}=0 \quad \text { (rotating }
$$

- $r=\mathrm{R}_{2}: \mathrm{V}=0$ and $\mathrm{U}=\mathrm{W}=0$

(fixed

outer cylinder)

For the upper and lower endwalls: $\mathrm{Z}=0$ and $\mathrm{Z}=\mathrm{H}$, $\mathrm{U}=\mathrm{V}=\mathrm{W}=0$

\section{Numerical Procedure}

Geometry creation and boundary conditions definition is done using axisymmetric 3D grids. The mesh is generated in structured quadrilateral cells. The analysis of the mesh-solution dependency on the number of grid cells is presented in Fig. 3, using the critical Taylor number matching to the first instability appearance versus mesh cells number.

This grid-refinement study led to adoption of a mesh with 1048576 cells, distributed in the radial (r), azimuthal $(\theta)$ and, axial (z) directions respectively.

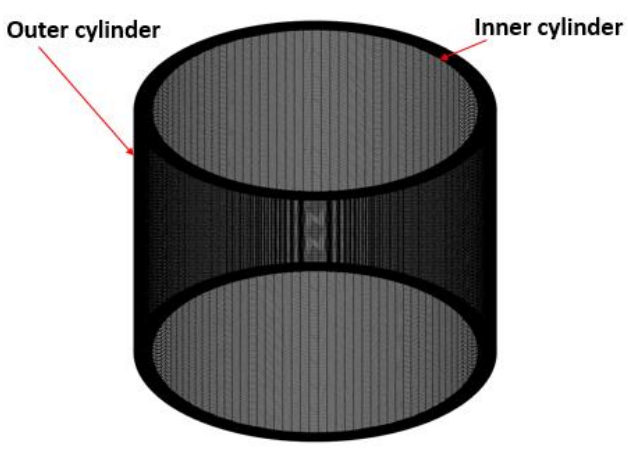

Fig. 2. Computational domain

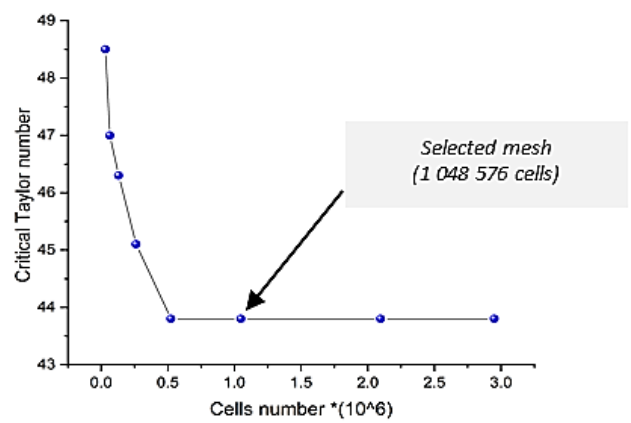

Fig. 3. Effect of the mesh size on the critical Taylor number

For numerical calculations the flow is considered three-dimensional and Navier-Stokes equations are solved in primitive formulation using Fluent software code based on the finite volume method discretization with a second order model for pressure and third order MUSCL scheme for velocity. This method is found to be convenient in such a way that the flow is considered laminar and can thus be computed without resolving turbulent scales. The analysis of the solution-mesh dependency on the grid is shown in Fig. 3. The critical Taylor number corresponding to the first instability settlement is depicted versus the mesh cells number varying from $3.2 \times 10^{4}$ to $2.94 \times 10^{6}$. The critical Taylor number ( $\mathrm{Ta}$ c, corresponding to the first instability triggering) is found for $1.048 \times 10^{6}$ mesh cells. The Implicit Pressure with Operator Division (PISO) algorithm is used for pressure-velocity coupling. Solution convergence is considered when all residuals are less than $10^{-4}$. The obtained numerical results are compared to literature. A maximum deviation of 5.9 $\%$ is found relatively to experiments of Bouabdallah (1980) and 3.8\% to those of Adnane et al. (2016).

Qualitative validation of the numerical simulations is executed using flow configuration comparison to experimental results of Khochmieder (1979) and Adnane et al. (2016), Fig.4. 
(a)

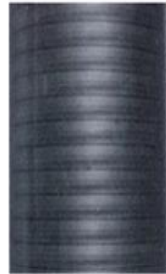

(a)

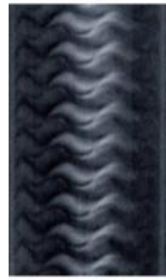

(b)

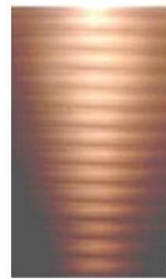

(b)

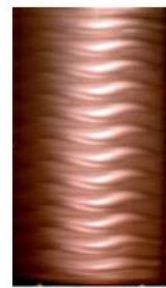

(c)

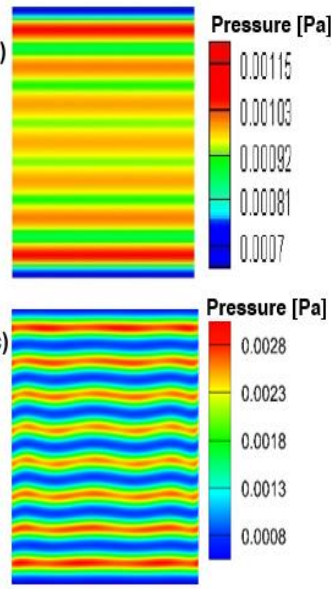

Fig. 4. Validation of the first and second instability appearance, $\mathrm{TVF}, \mathrm{Ta}_{\mathrm{c} 1}=43.8$ and $\mathrm{WVF}, \mathrm{Ta} \mathrm{a}_{2}=54$, characterized by pressure contours (a) experimental results of Khochmieder (1979), (b) experimental results of Adnan et al. (2016) and (c) present numerical study.

Table 1. Taylor number estimation of the first instability appearance.

\begin{tabular}{|c|c|c|c|c|c|}
\hline$N^{*}$ & Author & $\delta$ & $\eta$ & Tac1 & Difference, $\%$ \\
\hline 1 & Present work & 0.1149 & 0.8969 & 43.8 & 0 \\
\hline 2 & $\begin{array}{c}\text { Taylor, } \\
\text { Exp }\end{array}$ & 0.1366 & 0.8798 & 44.35 & 1.2 \\
\hline 3 & Andereck, Exp & 0.1326 & 0.8829 & 43.69 & 0.25 \\
\hline 4 & Coles, Exp & 0.1344 & 0.8815 & 42.51 & 3.03 \\
\hline
\end{tabular}

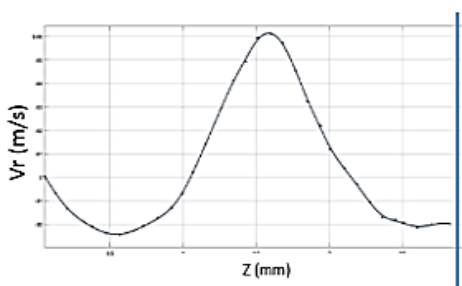

(a)

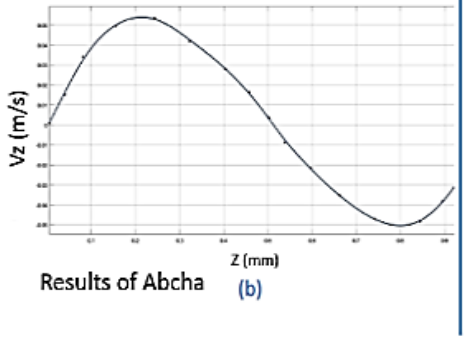

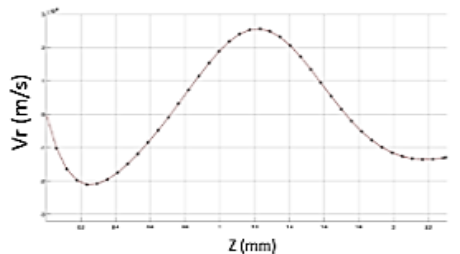

(c)

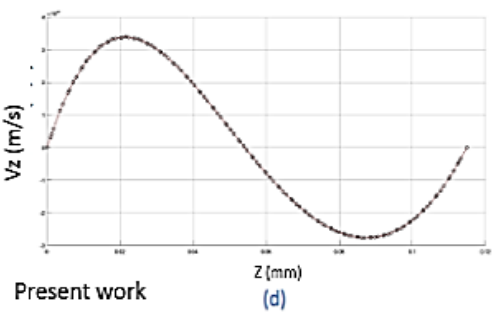

Fig. 5. Quantitative validation of the results by comparing with results of Abcha et al. (2008).

Table. 1 presents numerical estimation of the critical Taylor number relevant to first instability settlement (same non-dimensional numbers $\delta$ and $\eta$ ), compared to referenced works. Numerical results are in good agreement with experiments. Registered deviation is in between $0.25 \%$ to $3.03 \%$.

Another quantitative validation is made by comparing the present work data of radial and axial velocities along the axial position to those obtained by experiments of Abcha et al. (2008).

\section{RESULTS AND DISCUSSIONS}

\subsection{Flow behavior vortices of Ekman structuring stages}

The obtained results are presented for all the encountered flow regimes since the locally pumping Ekman cells, Fig. 6(a), in terms of pressure and streamline contours, ranging from low Taylor numbers $\left(\mathrm{Ta}=10^{-4}\right)$ corresponding to circular 


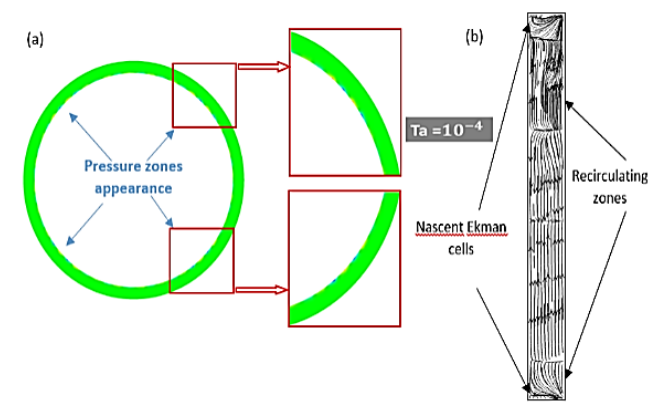

Fig. 6. System cross-section view: (a) Ekman vortices appearance with a phase shift of $(\pi / 2)$ at system endwalls in terms of pointwise pressure contours, (b) streamlines in the annulus for $\mathbf{T a}=$ $10^{-4}$.

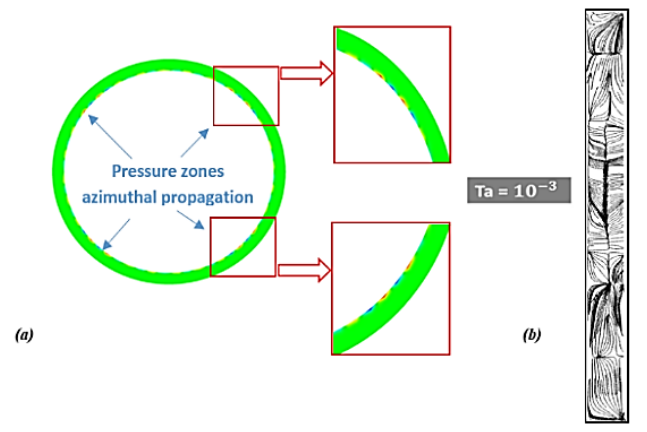

Fig. 7. System cross-section view: (a) Ekman vortices propagation with a phase shift of $(\pi / 2)$ at endwalls in terms of pressure contours (upper view), (b) Streamlines in the annulus for $\mathrm{Ta}=10^{-3}$ (front view).

Couette flow configuration $(\mathrm{CCF})$ to first instability onset $(\mathrm{TVF})$ at $\mathrm{Ta}_{\mathrm{c} 1}=43.8$. Appearance of Ekman cells signature is indicative of flow early transition triggering process towards the toroidal flow configuration (Taylor vortex flow TVF). The socalled wavy mode instability (WVF) is reached when increasing the Taylor number up to $\mathrm{Ta}_{\mathrm{c} 2}=54$.

For this flow configuration (rotating inner cylinder with the outer and endwalls at rest), there is continuous transition from featureless flow at very low Taylor number (from $\mathrm{Ta}=10^{-4}$ ) to a cellular flow induced by upper/lower endwalls and flow interaction. In fact, for this configuration the centrifugal force is balanced by the pressure gradient far from endwalls. Near these, however, the imbalance between inertial and viscosity forces induces a secondary Ekman pumping flow leading to Ekman vortices formation. These vortices signature is found to appear as localized pressure regions regularly separated with a phase shift of $(\pi / 2)$ for both top and bottom endwalls at $\mathrm{Ta}=10^{-4}$ (Fig. 6-(a)). In addition, the annulus streamlines reveal that flow structuration starts from both endwalls (Ekman cells pumping) giving rise to parallel ascendantdescendant flow meeting at the middle of the gap, Fig. 6-(b). When the Taylor number reaches the value $\mathrm{Ta}=10^{-3}$, a propagating process of pressure wave train zones initiates azimuthally on the inner cylinder surface which becomes totally covered when the Taylor number is in the range $\mathrm{Ta}=10^{-2}$ to $10^{-1}$, Figs. 7(a) and (b). It is worthwhile noting that flow structuration in the gap relies on several distinguished steps starting from Ekman cells appearance at the upper and lower endcaps, induced by cylinder lateral wall and endwalls layers interaction, followed by recirculating azimuthal zones contiguous to the upper and lower nascent Ekman cells, Fig. 6(b). These are separated by an intermediate ascendant-descendent flow (Fig. 6-(b)).

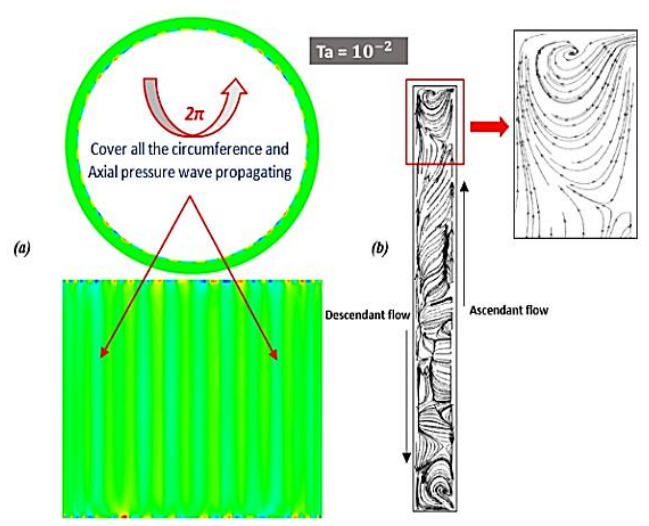

Fig. 8. (a) Complete appearance of the Ekman vortices in terms of pressure contours, (b) streamlines in the system annulus for $\mathrm{Ta}=10^{-2}$.

When slightly increasing the inner cylinder rotating rate, the pointwise pressure zones evolve by azimuthally spreading in an extremely slow mechanism to cover the whole gap along top and bottom endwalls circumferences at $\mathrm{Ta}=10^{-2}$, Fig. 8(a). In a parallel way, pressure train waves appear and propagate as longitudinal pressure iso-contours alternating between relatively high and low pressure values, (Fig. 8-(a)). These pressure wave trains are physically connected with the previously detected pointwise pressure zones; Fig. 8-(a). For the Taylor number in between $\mathrm{Ta}=10^{-2}$ to $\mathrm{Ta}=10^{-1}$, Ekman vortices take a circular shape close to the upper and lower system endwalls, Figs. 6-(b) and 7-(b), giving rise to Taylor vortices development leading to first and then second instability settlement.

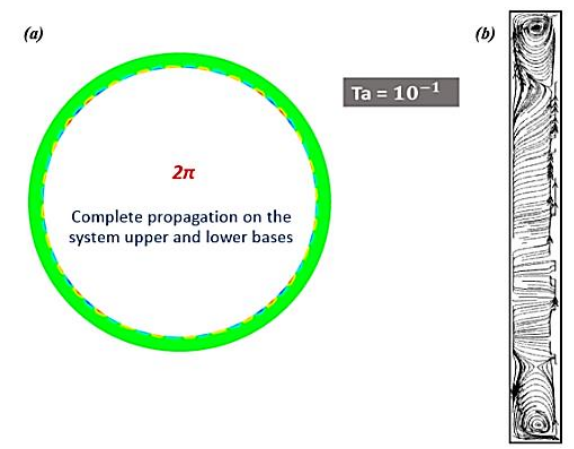

Fig. 9. System cross-section view: (a) Complete appearance of the Ekman vortices in terms of pressure contours, (b) streamlines in the annulus for $\mathrm{Ta}=10^{-1}$. 


\subsection{Flow characterization using streamlines}

It is sought here to shed light on the Taylor vortices genesis mechanism with description of all the intermediate "gestational" processes from "embryonic" stage up to complete structures onset. The flow characterization using streamlines in the $(\mathrm{r}, \mathrm{z})$ plane are adopted to visualize this flow evolution topology, Fig. 10. When increasing the inner cylinder rotation velocity, since the lowest reachable Taylor number to the first instability settlement, flow structuration in preparation to Ekman cells appearance, $\mathrm{Ta}=10^{-4}$, operates locally as previously reported, Figs. (6-7-8-9). Streamlines show weak Ekman cells with axial ascendantdescendent flow along the longitudinal mid-gap plane from bottom and top, respectively, to center. Axial pressure waves give rise to nascent Ekman vortical layers induced by a counterbalance between centrifugal force, resulting from inner cylinder rotation, and pressure force. With a further slight increase in Taylor number, from $\mathrm{Ta}=10^{-2}$ to $\mathrm{Ta}=5$, Ekman vortices evolve to a completely circular shape since $\mathrm{Ta}=10^{-2}$. These Ekman vortices are thought to be responsible for the upcoming flow structuring process starting at $\mathrm{Ta}=$ 15. Increasing the Taylor number up to $\mathrm{Ta}=30$ leads to propagation of Taylor vortices filling all the gap width from endwalls to gap center, indicative of the first instability settlement at $\mathrm{Ta}=$ 43.8 .

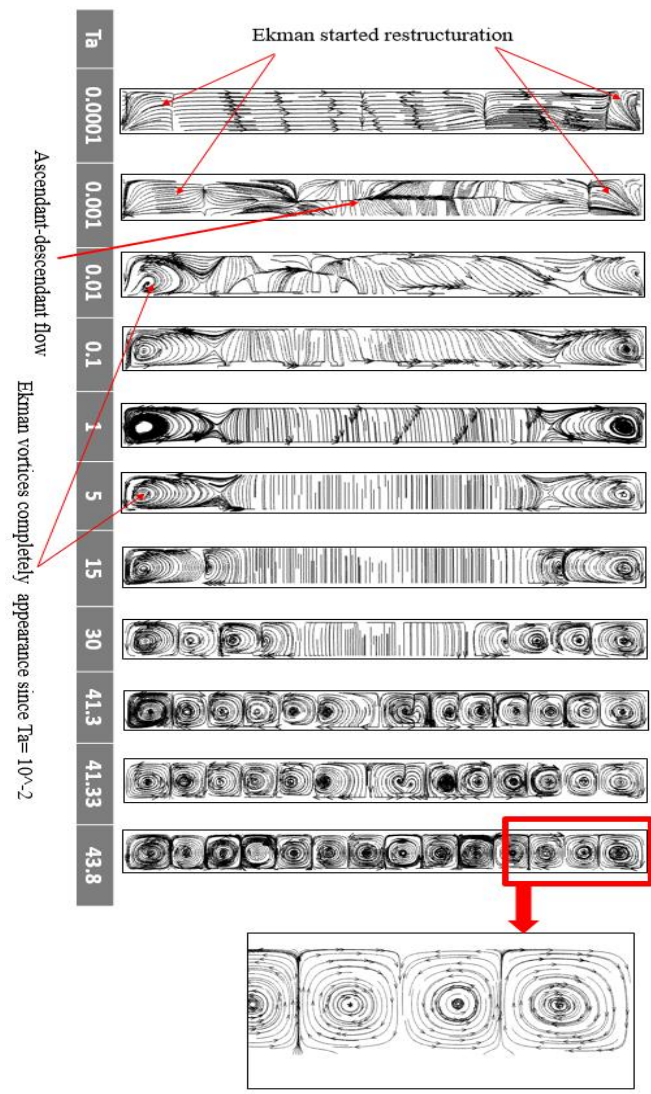

Fig. 10. Flow characterization using streamlines configuration in $(r, z)$ plan.
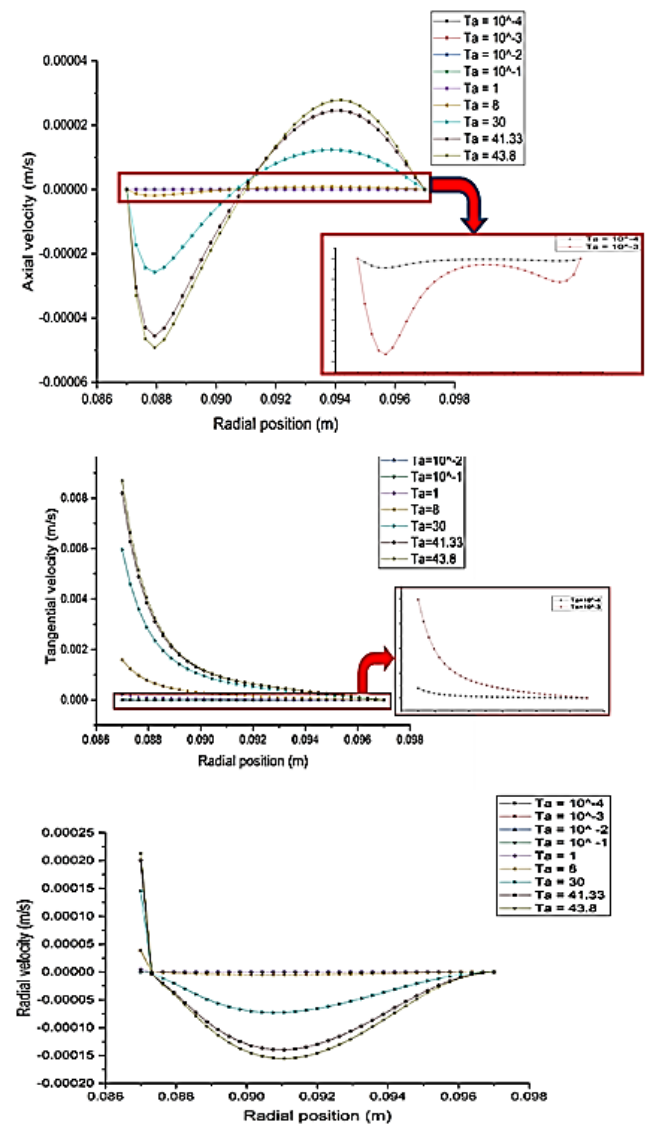

Fig. 11: Axial, Tangential and radial velocity variation in the gap for different Taylor numbers.

Figure 11 depicts variation of axial, tangential and radial velocities in the gap for different Taylor numbers. It is noted that for low Taylor numbers $(\mathrm{Ta} \leq 10)$ the flow is laminar and steady based on the resulting velocities negligible value. A Ta $=41.33$, Taylor vortices propagate axially in the whole gap, and when Ta becomes equal to 43.8 the first instability is settled and notable variations in tangential and radial velocities are registered.

\subsection{Flow characterization of the first and second instability triggering (TVF, WVF):}

Genesis mechanism starts by pressure gradient inclusions appearing at four localized regions separated by $90^{\circ}$ angles. Then, azimuthally propagate to meet when Ta $10^{-2}$ to $10^{-1}$, at this stage of the flow, Ekman vortices initiate alternating axial pressure bands (an axial pressure wave propagating along axial-azimuthal directions ) along the inner wall of the outer cylinder. These waves become clearly pronounced and schematically visible at $\mathrm{Ta}=$ 0.01 , Fig. 12 (b). From $\mathrm{Ta}=1$ the Taylor vortices formation mechanism triggers consecutively to the Ekman vortices complete formation mechanism launched much earlier since $\mathrm{Ta}=10^{-4}$ to $\mathrm{Ta}=10^{-2}$. The Taylor stationary wave starts then propagating in the axial direction from system endwalls to meet at the system mid-height at $\mathrm{Ta}=43.8$. This is 
achieved on expenses of the successive pressure wave trains, which gradually vanish in favor of the nascent azimuthal wave. The process stops with complete disappearance of the pressure wave trains at $\mathrm{Ta}=43.8$. The cylindrical cavity is then entirely filled with piled toroidal vortices corresponding to the first instability settlement commonly known as the steady Taylor wave (TVF), Fig. 12.

\section{Fig. 12. Flow regimes from (CCF) to (TVF)}
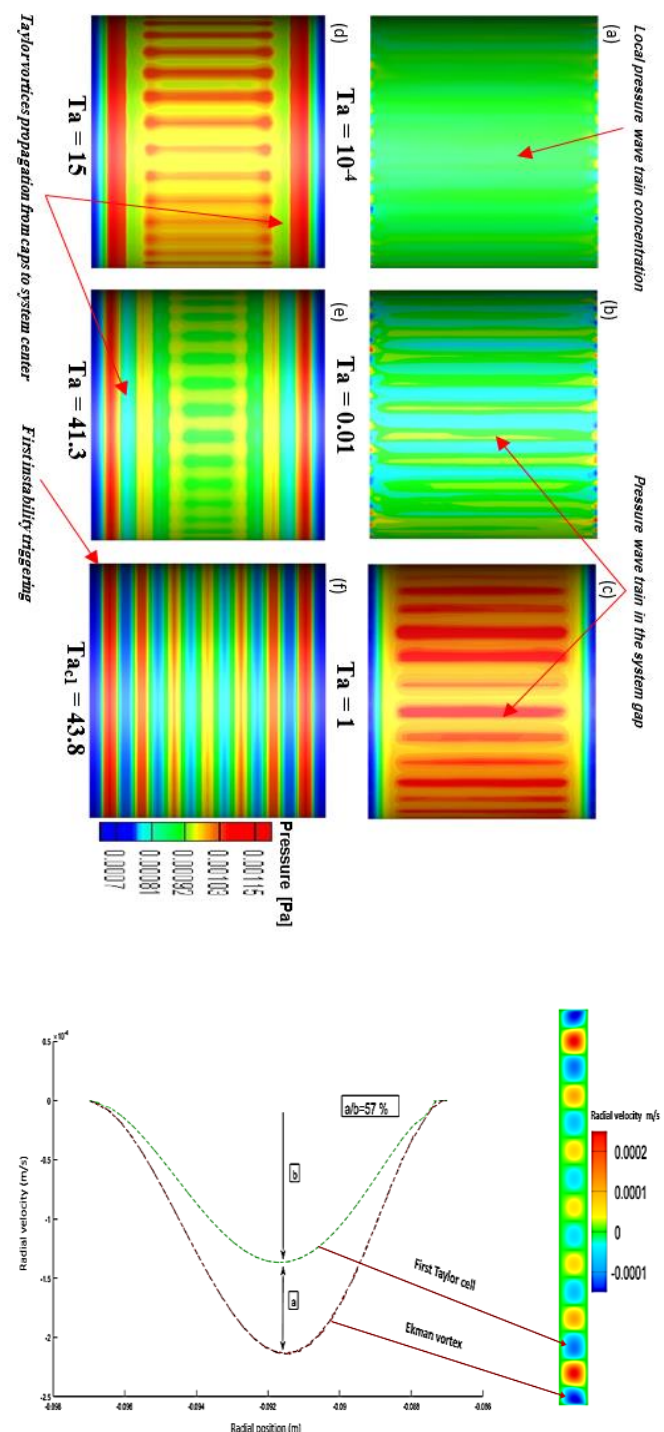

Fig. 13. Evolution of the radial velocity in the annular space of Ekman vortex and the first appeared Taylor cell.

Figure 13 depicts evolution of the radial velocity in the annular space for Ekman vortex and the first appeared Taylor cell. It is seen that the Ekman cell has a radial velocity $40 \%$ to $60 \%$ larger comparatively to the contiguous Taylor cell, as reported by Czarny et al. (2003). This is quantitatively indicative of the first instability settlement. Qualitatively, TVF mode accomplishes when the gap volume is entirely filled with superposed toroidal vortices along the axial direction at $\mathrm{Ta}_{\mathrm{c} 1}=43.8$. As the inner cylinder rotation velocity increases, this flow (TVF) evolves to an unsteady configuration commonly reported as wavy mode, WVF, Fig. 14.

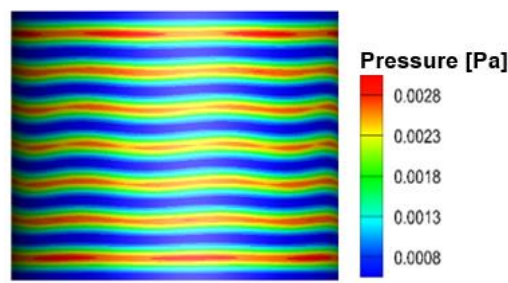

Tac2 $=54$

Fig. 14. Wavy mode flow configuration (2nd instability WVF).

The axisymmetric Taylor flow becomes then unstable resulting in an unsteady flow characterized by a wavy motion (azimuthal wave) superimposed on Taylor's vortices. The set of these vortices undulate in the axial direction with no phase lag indicating the second instability settlement characterized by unsteady Taylor waves at the critical Taylor number value, $\mathrm{Ta}_{22}=54$. This is the so-called wavy mode where the toroidal vortex centerline is driven in a double axial and tangential dynamics.

The flow behavior in terms of velocity components; radial, axial and tangential is showed in Figs. 15 and 16 for the TVF and WVF regimes respectively. An analysis of the radial flow topology shows the counter-rotating piled Taylor configuration for both the TVF and WVF flow regimes. In addition, the axial velocity component presents the symmetry of TVF regime. The tangential velocity contours show the inflow and outflow positions along the gap length between the two coaxial cylinders and the boundary layer width evolution on the inner and outer cylinders. It is also noted that $\mathrm{Vr}$ and $\mathrm{Vz}$ are similar for this flow system, while $\mathrm{V}_{\theta}$ clearly highlights existence of fluctuations on the rotative wall.

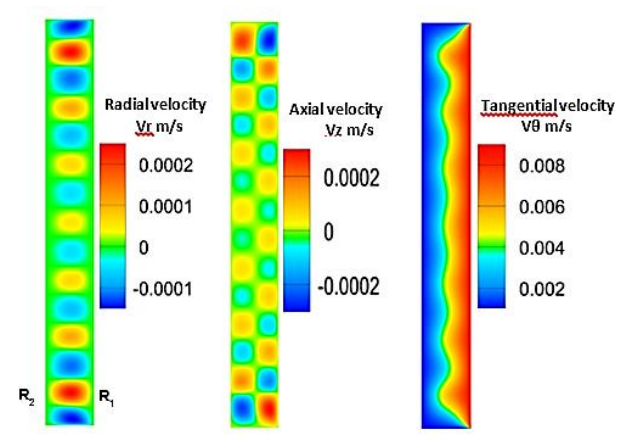

Fig. 15. TVF velocity components contours. 


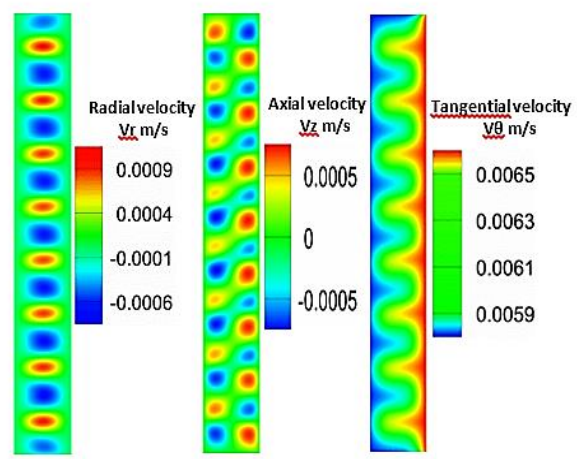

Fig. 16. WVF velocity components contours.

\section{CONCLUSION}

A numerical simulation is carried out and devoted to characterizing the mechanism genesis of Ekman and Taylor-Couette flow. The study is focused on the flow structuring process from the early stages where the Ekman vortices generate at the system endcaps. It is established that the Ekman cells appear in local positions with a phase shift of $(\pi / 2)$ at a Taylor number as low as $10^{-4}$. For $10^{-4} \leq \mathrm{Ta} \leq 10^{-2}$, the flow evolves as ascendant-descendant in presence of recirculating regions, a weak increasing in Taylor number lead to the subsequent stage in flow structuring process (Ekman vortices genesis). These vortices signature are already present at $\mathrm{Ta}=10^{-2}$.

The pointwise cells spread along the azimuthal direction leading to Taylor vortices formation on the pressure wave expenses. This constructing mechanism is triggered and piloted by intricate interacting pressure waves propagating in the axialazimuthal plane inducing Ekman then Taylor vortices formation. The latter propagate from system endcaps to gap center, inducing pressure wave trains progressive vanishing. The genesis mechanism ends by giving birth to TVF then WVF regimes at $\mathrm{Ta}=$ 43.8 and 54 respectively.

\section{REFERENCES}

Abcha, N., N. Latrache, F. Dumouchel and I. Mutabazi (2008). Qualitative relation between reflected light intensity by Kalliroscope flakes and velocity field in the Couette- Taylor flow system.Experiments in Fluids (45), 85-94.

Abdelali, A., H. Oualli and A. Bouabdallah (2019). Experiment and numerical simulation of Taylor-Couettefow controlled by oscillations of inner cylinder cross section. Journal of the Brazilian Society of Mechanical Sciences and Engineering 41, 259.

Adnane, E. and A. Bouabdallah (2016). Experimental Study of the Laminar-Turbulent Transition in a Tilted Taylor-Couette System Subject to Free Surface Effect. Journal of Applied Fluid Mechanics 9(3), 1097-1104.
Ahlers, G. and D. S. Cannell (1983). Vortex-front propagation in rotating Couette-Taylor flow. Physical Review Letters 50, 1583-1586.

Andereck, C. D., S. S. Liu and H. L. Swinney (1986). Flow regimes in a circular Couette system with independently rotating cylinders. Journal of Fluid Mechanics 164, 155-183.

Bouabdallah, A. (1980). Instabilités et turbulence dans l'écoulement de Taylor-Couette, $\mathrm{PhD}$ Thesis, INPL, France.

Coles, D. (1965). Transition in circular Couette flow. Journal of Fluid Mechanics 21, 385-425.

Couette, M. M. (1890). Etudes sur le frottement des liquides. Annex of Chemistry Physics 433.

Czarny, O., E. Serre, P. Bontoux and R. M. Lueptow (2002). Spiral and wavy vortex flows in short counter-rotating Taylor-Couette cells. Theoret. Comput. Fluid Dynamics 16, 5-15.

Czarny, O., E. Serre, P. Bontoux and R. M. Lueptow (2003). Interaction between Ekman pumping and the centrifugal instability in TaylorCouette flow. Physics of Fluids 15, 467-477.

Czarny, O., E. Serre, P. Bontoux and R. M. Lueptow (2004). Ekman vortices and the centrifugal instability in counter-rotating cylindrical Couette flow. Theoretical and Computational Fluid Dynamics 18, 151-16.

Denis, M., S. Eric and R. M. Lueptow(2014). Mechanisms for the transition to waviness for Taylor vortices. Physics of Fluids 26, 094102

Fenstermacher, P. R. and H. L. Swinney (1979). Dynamical instabilities and the transition to chaotic Taylor vortex flow. Journal of Fluid Mechanics 94, part 1, 103-128.

Goharzadeh, A. and I. Mutabazi (2001). Experimental characterization of intermittency regimes in the Couette-Taylor system. European Physical Journal B 19, 157-162.

Gollub, J. P. and H. L. Swinney (1975). Onset of turbulence in a rotating fluid. Physical Review Letters 35, 927-930.

Koschmeider, E. L. (1993). Bénard cells and Taylor vortices. Journal of Fluid Mechanics 253, 722 723.

Lücke, M., M. Mihelcic and K. Wingerath (1985). Front propagation and pattern formation of Taylor vortices growing into unstable circular Couette flow. Physical Review A 31, 396-409.

Ludwieg, H. (1964). Experimentelle Nachprüfung des Stabilitäts theorien für reibungsfreie StrömungenMitschraubenlinienförmigen Stromlinien. Z. Flugwiss 12, 304-309.

Margules, M. (1881). Uber die bestimmung des reibungs-und gleitungs-coefficient, Wiener Berichte (second series) 83, 588-602.

Oualli, H., S. Hanchi and A. Bouabdallah (2013). Taylor-Couette flow control using the outer 
M. Khirennas et al. / JAFM, Vol. 15, No. 1, pp. 129-137, 2022.

cylinder cross-section variation strategy. European Physical Journal Applied Physics 61,11102 .

Pfister, G. and I. Rehberg (1981). Space-dependent order parameter in circular Couette flow transitions. Physical Letters 83A, 19-22.

Rayleigh, L. (1916). On the dynamics of revolving fluids. proceedings of the royal society of London, A, 148-154.

Serre, E., M. A. Sprague and R. M. Lueptow (2008).Stability of Taylor-Couette flow in a finite-length cavity with radial throughflow. Physics of Fluids 20, 034106.

Stockes, G. G. (1880). Mathematical and physical papers. Cambridge. U. P, England.

Taylor, G. I. (1923). Stability of a viscous liquid contained between two rotating cylinders. Philosophical Transactions of the Royal Society A. London, 223, 289-343. 